\title{
Violencia familiar, depresión y autoestima en confirmantes de la Parroquia Jesús Vida y Paz en el Perú
}

\section{Family violence, depression and self-esteem in confirmations of the Jesús Vida y Paz Parish in Peru}

DOI: $10.46932 /$ sfjdv2n5-085

Received in: Oct 1st, 2021

Accepted in: Dec 30th, 2021

\author{
Carmín Nelly León Tarazona \\ Mg. Administración de la Educación \\ Universidad César Vallejo \\ Av. Alfredo Mendiola 6232, Los Olivos 15314 \\ Correo: misscarminleon@gmail.com \\ Ángel Iván Ruiz Mesías \\ Mg. Educación \\ Universidad Inca Garcilaso de la Vega \\ Jr. Saco Oliveros No 310 Sta. Beatriz - Lima \\ Correo: ruizmesias@gmail.com \\ Karla Robalino Sánchez \\ Mg. Investigación y Gestión del Patrimonio Histórico Artístico y Cultural \\ Universidad de Murcia \\ Campus La Merced, 30071, Murcia \\ Correo: karlarosa0407@gmail.com

\section{Rosa Diana Pedroza San Miguel} \\ Dr. Psicología \\ Universidad César Vallejo \\ Av. Alfredo Mendiola 6232, Los Olivos 15314 \\ Correo: rosa.pedroza29@gmail.com
}

\section{RESUMEN}

Esta investigación tuvo como propósito determinar la influencia de la violencia familiar y depresión en la autoestima en confirmantes de la Parroquia Jesús Vida y Paz del distrito de San Juan de Lurigancho, 2021. Este estudio fue de tipo básica, se usó el diseño no experimental, de corte transversal, correlacional causal y enfoque cuantitativo. La muestra estuvo conformada por 140 personas de ambos sexos. El estadístico aplicado fue la regresión logística multinomial. Los resultados mostraron que $47.1 \%$ de la muestra tenía baja autoestima, $24.3 \%$ depresión grave y $4.3 \%$ padecía violencia familiar. Respecto al sexo, los varones evidenciaron mejores resultados en cuanto a la autoestima, menores niveles de depresión y no hubo diferencia significativa entre los niveles de violencia familiar. El ajuste del modelo además de ser adecuado explica en un $60.6 \%$ la probabilidad de ocurrencia de las categorías de la variable dependiente. Se concluyó que la violencia familiar y la depresión influyen en la autoestima en confirmantes de la Parroquia Jesús Vida y Paz del distrito de San Juan de Lurigancho, 2021.

Palabras clave: Autoestima, COVID - 19, depresión y violencia familiar. 


\begin{abstract}
This research aimed to determine the influence of family violence and depression on self-esteem in young confirming from the Jesus Vida y Paz Parish of the San Juan de Lurigancho, 2021. This study was of a basic type, the non-experimental design was used, cross-sectional, causal correlational and quantitative approach.The sample consisted of 140 people of both sexes. The applied statistic was multinomial logistic regression. The results showed that $47.1 \%$ of the sample had low self-esteem, $24.3 \%$ had severe depression and $4.3 \%$ suffered family violence. Regarding sex, men showed better results in terms of selfesteem, lower levels of depression but there was no significant difference between levels of family violence. The adjustment of the model, in addition to being adequate, explains by $60.6 \%$ the probability of occurrence of the categories of the dependent variable. It was concluded that family violence and depression influence self-esteem in those confirmed by the Jesus Vida y Paz Parish of the San Juan de Lurigancho district, 2021.
\end{abstract}

Keywords: Self-esteem, COVID - 19, depression and family violence.

\title{
1 INTRODUCCIÓN
}

Vivimos en una situación de pandemia sin precedentes. La población mundial se ha visto sometida a un aislamiento social e inmovilización el cual incrementó diversas problemáticas y de entro de ellas, la violencia familiar. La disposición gubernamental de impedimento de salir de las casas generó que víctimas y victimarios, convivan día a día. De acuerdo con el diario El Comercio (2020) el Ministerio Público registró 3763 personas detenidas por agresiones graves a mujeres en todo el país, asimismo reportaron 7 feminicidios y 17 tentativas, por otro lado, el Ministerio de la Mujer y Poblaciones Vulnerables (MIMP) ha registrado 12 feminicidios y 20 tentativas desde el 16 de marzo hasta el 5 de mayo. Esta diferencia se debe lamentablemente, a la diferencia en la metodología para registrar los cambios.

Una de las jurisdicciones que ha sido mencionada en reiteradas ocasiones como un lugar en donde la violencia familiar está muy presente es en el distrito de San Juan de Lurigancho. El noticiero Radio Programas del Perú (2017), refirió que el Ministerio Público reportó que 12 distritos de Lima registraron, en los últimos 8 años, los índices más altos de feminicidio con un total de 184 casos, el 22\% sucedió en San Juan de Lurigancho, convirtiéndolo en el distrito donde hay más violencia. El Ministerio de la Mujer y Poblaciones Vulnerables (MIMP) a través del Programa Nacional para la Prevención y Erradicación de la Violencia contra las Mujeres e Integrantes del Grupo Familiar - Aurora (2019), refirió que el informe de los cinco Centros de Emergencia Mujer establecidos en dicho distrito, reportaron desde enero hasta abril de 2019, un total de 1238 casos atendidos por violencia familiar, de los cuales el $89 \%$ fueron agresiones contra las mujeres. Es así que nos planteamos la siguiente problemática: ¿cómo la violencia familiar y la depresión influyen en la autoestima en confirmantes de la Parroquia Jesús Vida y Paz del distrito de San Juan de Lurigancho, 2021?. 
Del cuestionamiento referido se desprende que la justificación de nuestra investigación es hacer un análisis que invite a la comunidad científica a debatir sobre la influencia de la violencia familiar y la depresión en la autoestima de las personas, más aún bajo el contexto de una pandemia que hizo que víctimas y victimarios estén confinados en un mismo espacio, daño colateral de la decisión gubernamental para hacer frente a la COVID - 19.

Es por ello por lo que como objetivo general planteamos determinar cómo la violencia familiar y la depresión influyen en la autoestima en confirmantes de la Parroquia Jesús Vida y Paz del distrito de San Juan de Lurigancho, 2021. De ello se desprende como objetivos específicos: (1) determinar cómo la violencia familiar y la depresión influyen en la dimensión sí mismo general de la variable autoestima en confirmantes de la Parroquia Jesús Vida y Paz del distrito de San Juan de Lurigancho, 2021; (2) determinar cómo la violencia familiar y la depresión influyen en la dimensión social de la variable autoestima en confirmantes de la Parroquia Jesús Vida y Paz del distrito de San Juan de Lurigancho, 2021 y; (3) determinar cómo la violencia familiar y la depresión influyen en la dimensión familiar de la variable autoestima en confirmantes de la Parroquia Jesús Vida y Paz del distrito de San Juan de Lurigancho, 2021.

\section{MARCO TEÓRICO}

En referencia a los trabajos previos internacionales; Dostanic, et al. (2021) determinaron la relación entre la depresión, ansiedad y violencia de pareja en mujeres de Serbia. La muestra la conformaron 104 mujeres cuyos esposos recibían tratamiento por consumo del alcohol. Los instrumentos aplicados el inventario de depresión de Beck, el inventario de ansiedad de Beck, el test de identificación en desorden de abuso de alcohol y la escala de tácticas de conflicto. Los estadísticos utilizados fueron el chi-cuadrado y el análisis de regresión logística multinomial. Los resultados mostraron que los el 33.5\% de la muestra tenían depresión moderada y el 34.6\% a nivel severo, asimismo que la violencia bajo los efectos del alcohol incrementa en $25 \%$ la probabilidad de padecer de depresión moderada y $220 \%$ en depresión severa y en cuanto a la ansiedad, ésta incrementa en 33\% la probabilidad de tener ansiedad en nivel severo. Se concluyó que la violencia de pareja bajos los efectos del alcohol se relacionan con la depresión y ansiedad en las mujeres.

Chahid, et al (2020) buscaron determinar la relación entre la depresión, autoestima, ansiedad e intentos de suicidio. El estudio fue correlacional y la muestra la conformaron 200 estudiantes entre los 15 y 21 años de Marruecos. Los instrumentos utilizados fueron el inventario de depresión de Beck, el cuestionario de evaluación de ansiedad y la escala de autoestima de Rosenberg. El estadístico aplicado fue el análisis de regresión múltiple y la $\mathrm{r}$ de Pearson. Los resultados mostraron que el 30\% de la muestra padecía, $73.5 \%$ tenían ansiedad y el $70 \%$ poseía una baja autoestima; en todos los casos las mujeres mostraron tener mayor depresión, mayor ansiedad y menor autoestima en comparación de los varones. La 
autoestima se correlacionaba de manera negativa con la depresión $(r=-.308)$, de igual manera con la ansiedad $(\mathrm{r}=-461)$; asimismo la ansiedad se correlacionada de manera positivamente con la depresión $(\mathrm{r}$ $=.427$ ). Respecto a los resultados de la regresión múltiple, las tres variables predecían en un $10.1 \%$ la probabilidad de intento de suicidio. Se concluyó que la ansiedad, depresión y autoestima son factores que influyen en los intentos de suicidio.

En referencia a los trabajos previos nacionales, Astocondor, et al (2019) determinaron el nivel de la influencia de la depresión en cuanto a la ideación suicida de los alumnos en la selva de Perú. En cuanto al diseño de investigación fue transversal y analítico. La muestra la conformaron 431 estudiantes de la Universidad Nacional de Ucayali, quienes fueron debidamente elegidos mediante un muestreo de tipo no probabilístico y por conveniencia. El instrumento utilizado y validado para el Perú fue la escala de depresión de Birleson y de Beck, respectivamente. El estadístico utilizado fue la prueba chi cuadrado. Los resultaron evidenciaron que las mujeres $(57.1 \%)$ tienen mayor depresión que los varones (42.9\%) y que existe una gran relación entre la ideación suicida y la depresión (valor $\mathrm{p}<.01$ ). Se concluyó que existe relación entre las variables tales como la ideación suicida y la depresión, que está fundamentada por algunas percepciones y conductas que señalan grandes problemas de la esfera mental.

Llanos y Ponce (2019), determinaron la relación entre la variable depresión y la carencia de cohesión al tratamiento contra la tuberculosis en pacientes del Hospital Nacional Dos de Mayo durante el año 2017. El estudio fue observacional, descriptivo y prospectivo. La muestra estuvo conformada 28 varones y 22 mujeres cuyo diagnóstico fue de tuberculosis pulmonar y con estado nutricional normal más frecuente. En cuanto a los instrumentos se utilizaron el cuestionario PHQ-9 para conocer el nivel de depresión, y el cuestionario Morisky-Green para calcular la adherencia al tratamiento de este. Los resultados indicaron que el promedio de faltas fue $6.5 \%$. Un $38 \%$ de pacientes fueron seleccionados como no adherentes y la presencia de la depresión fue en un $76 \%$. Se obtuvo una relación estadísticamente significativa de ( $>>0.05)$ entre los diversos niveles de adherencia y de depresión, según los instrumentos anteriormente mencionados. Por otro lado, se evidenció una asociación entre adherencia y tener más de cinco faltas durante la primera etapa del tratamiento. Se concluyó que existe una relación notable entre el nivel de adherencia al tratamiento contra la tuberculosis y la presencia de depresión. De igual manera se evidenció la gran elevada frecuencia de no adherencia al tratamiento en la primera fase y la frecuencia elevada de síntomas que sugestionan a la depresión y la asociación entre adherencia y haber tenido 5 o más faltas.

Existen diversos modelos teóricos sobre violencia familiar, Ramírez (como se citó en Gonzales, 2017) refiere que hay un modelo biológico que explica la violencia como parte de la estructura del ser humano. Si bien, el hombre como especie ha sobrevivido usando la violencia se tiene la concepción de que es por cumplir un rol protector, sin embargo, eso no da una explicación satisfactoria de porque algunos 
varones no son violentos, aunque sean más fuertes que sus parejas e incluso algunas mujeres son violentas con los varones.

Perrone (2012, como se citó en Losada y Caruso, 2021) refirió que hay tres tipos de violencia en función a la posición en la que se encuentran las personas implicadas: 1) La violencia agresión, se da entre personas que tiene el mismo posiciones equivalentes, es decir no hay desventaja en la relación confrontante. La violencia se exhibe como agresión física y verbal mediante intimidaciones, gritos, golpes, etc., 2) violencia castigo, en donde uno de los individuos está en desventaja debido a que tiene una relación complementaria con el agresor. La víctima, que presenta baja autoestima y se desprecia, presenta dificultades para defenderse y se ve obligado sin poner oposición, a aguantar el ataque del agresor quien se aprovecha de las debilidades llegando a un sometimiento e incluso dependencia patológica y; 3 ) violencia represalia, en este caso, la persona en posición desfavorable empieza a resistirse ante la agresión a pesar de la desproporción que existe entre ellos, pues espera que la situación se revierta.

En cuanto a la segunda variable depresión, existen varias concepciones. Según, Beck (como se citó en Ocampo, et al., 2017) trata de explicar que la cognición explica este estado. Está estructurado en un modelo de labilidad ante el estrés, con pensamientos distorsionados y que hacen que la percepción, codificación e interpretación de sí mismo, de su entorno y del futuro sea negativo.

Para Villegas (2021), la depresión se plantea desde un contexto de filosofía existencial en la que el mundo es el entorno en donde se da la proyección del propio yo con relación a la interacción con lo demás. Desde dicha perspectiva, tenemos lo siguiente: 1) fallo ontológico, en donde el ser humano considera ilegítima su propia existencia, es así que su ser es eliminado de manera directa y por ello se producen los suicidios; 2) exclusión social, las personas que padece de depresión es porque se sienten perseguidos, excluidas, deshonradas y humilladas en sus relaciones interpersonales tanto públicas como privadas y; 3) fracaso existencial en donde la persona no encuentra sentido a su propia existencia que conlleva a ideas de muerte y suicidio.

En cuanto a la variable autoestima, Diez (2021) refirió que diversos estudios demuestran que una adecuada autoestima se correlaciona de manera inversa con dificultades psicosomáticas, depresión, ansiedad estado - rasgo, ideación paranoide, problemas académicos, estrés en estudiantes universitarios y conductas antisociales; es decir que a medida la autoestima es más alta, ayuda a tener menor intensidad en problemas de salud mental. Lo mismo expresó Estrada, et al., (2021), añadieron que tener un bajo nivel de autoestima favorece la presencia trastornos de alimentación, mala percepción física, sentirse inferior a los demás, aislamiento social, consumo de drogas, entre otros.

Paucar y Barboza (2018) afirmaron que la autoestima tiene cuatro componentes que la conforman y son: 1) componente cognitivo, hace referencia a los rasgos con los que una persona de describe a sí mismo y que implica modificar los pensamientos negativos por unos positivos que impacten en mi 
descripción personal; 2) componente afectivo, en la que tenemos sentimientos que son favorables o no de nosotros mismos; 3) componente conductual, en donde se presenta nuestra autoafirmación como personas pero a su vez valoramos el reconocimiento de los demás por nosotros y; 4) componente actitudinal, es el sistema elemental en la que organizamos las experiencias de nuestro propio yo.

\section{MÉTODO}

De acuerdo con Vara (Espinoza, 2016), esta investigación corresponde al tipo básica y su propósito es ampliar los conocimientos ya establecidos en la que se estudia la relación entre las variables en una realidad para entenderlo desde una nueva perspectiva.

El diseño de investigación conforme con Alarcón (Fernández, 2019), el diseño de esta investigación es no experimental, ya que no manipulamos ninguna de las variables; asimismo es de tipo correlacional causal en la medida que analizamos la relación entre las variables y de corte transversal, dado que los datos se recolectan en un solo momento.

El enfoque de investigación para esta investigación, se usó el enfoque cuantitativo. De acuerdo con Sánchez, et al (Rosas, 2019) en este enfoque se recolectan los datos y posteriormente se analizan para responder preguntas y probar las hipótesis aplicando la estadística.

La población estuvo constituida por 140 personas de ambos sexos y la muestra es la misma cantidad. Para este estudio, se aplicaron tres instrumentos para recolectar los datos, siendo el primero de ellos una escala de autoestima, el segundo el cuestionario sobre violencia familiar y el tercero el inventario de depresión, a través de la escala de Likert de 5 puntuaciones.

Luego de que se exporten los datos, se ordenaron y se procesaron mediante el programa informático SPSS v. 25 con el objetivo de contrastar las hipótesis del estudio, lo que nos permitirá determinar la influencia de la violencia familiar y la depresión en la autoestima confirmantes de la Parroquia Jesús Vida y Paz del distrito de San Juan de Lurigancho.

Finalmente, se procederá al análisis estadístico mediante la aplicación de estadística descriptiva tal como uso de tablas de frecuencias, porcentajes y figuras asimismo usaremos estadística inferencial mediante la Chi cuadrada y la R2 de Nagelkerke. Quevedo (2011) refirió que la Chi cuadrada, es un estadístico que evaluar la distribución teórica respecto a la distribución real de los datos obtenidos en una muestra mientras que la R2 de Nagelkerke, Díaz (2017) intenta estimar en qué grado la o las variables independientes pueden explicar la varianza en los resultados de la variable dependiente. 


\section{RESULTADOS}

Sobre los resultados inferenciales tenemos:

Tabla 1 . Influencia de las variables independientes en la variable dependiente y dimensiones.

\begin{tabular}{|c|c|c|c|c|c|}
\hline Hipótesis & $\begin{array}{l}\text { Variables * } \\
\text { Correlación }\end{array}$ & $\begin{array}{l}\text { Pseudo R } \\
\text { cuadrado } \\
\text { Nagelkerke }\end{array}$ & Chi-cuadrado & $\mathrm{gl}$ & Sig. \\
\hline $\begin{array}{l}\text { Hipótesis } \\
\text { general }\end{array}$ & $\begin{array}{c}\text { Violencia familiar y } \\
\text { depresión* } \\
\text { Autoestima }\end{array}$ & ,606 & 8,130 & 4 &, 000 \\
\hline $\begin{array}{l}\text { Hipótesis } \\
\text { específica-1 }\end{array}$ & $\begin{array}{c}\text { Violencia familiar y } \\
\text { depresión* Sí mismo } \\
\text { general }\end{array}$ & ,587 & 11,297 & 4 & ,000 \\
\hline $\begin{array}{l}\text { Hipótesis } \\
\text { específica-2 }\end{array}$ & $\begin{array}{l}\text { Violencia familiar y } \\
\text { depresión* Social }\end{array}$ & ,532 & 14,513 & 4 & ,000 \\
\hline $\begin{array}{l}\text { Hipótesis } \\
\text { específica-3 }\end{array}$ & $\begin{array}{l}\text { Violencia familiar y } \\
\text { depresión* Familiar }\end{array}$ & ,629 & 5,403 & 4 & ,000 \\
\hline
\end{tabular}

*. La correlación es significativa en el nivel 0,05 (bilateral).

De los resultados anteriores comprobamos que entre las variables violencia familiar y depresión con la autoestima, existe una influencia considerable al obtener un porcentaje de influencia de Nagelkerke del 60,6\%. Se obtuvo el valor de Chi-cuadrado de 8,130 que por ser menor al valor del Chi-cuadrado teórico de 21,03 se estableció que es el modelo adecuado para el ajuste de los datos. Así mismo esta influencia está reforzada por el valor de significancia obtenido de $\mathrm{p}(\mathrm{sig})=0.000$ y es menor de 0.05 ; se rechaza la hipótesis nula y se acepta la alterna, demostrando que si hay influencia entre las variables independientes con la variable autoestima.

De acuerdo con la hipótesis específica 1, establecimos una influencia de las variables violencia familiar y depresión en la dimensión sí mismo general de la autoestima y se afirma que existe una influencia considerable al obtener un porcentaje de influencia de Nagelkerke del 58,7\%. Se obtuvo el valor de Chi-cuadrado de 11,297 que por ser menor al valor del Chi-cuadrado teórico de 21,03 se estableció que es el modelo adecuado para el ajuste de los datos. Así mismo esta influencia está reforzada por el valor de significancia obtenido de $\mathrm{p}(\mathrm{sig})=0.000$ y es menor de 0.05 ; se rechaza la hipótesis nula y se acepta la alterna, demostrando que si hay influencia entre las variables independientes con la dimensión sí mismo general.

De acuerdo con la hipótesis específica 2, establecimos una influencia de las variables violencia familiar y depresión en la dimensión social de la autoestima y se afirma que existe una influencia considerable al obtener un porcentaje de influencia de Nagelkerke del 53,2\%. Se obtuvo el valor de Chicuadrado de 14,513 que por ser menor al valor del Chi-cuadrado teórico de 21,03 se estableció que es el 
modelo adecuado para el ajuste de los datos. Así mismo esta influencia está reforzada por el valor de significancia obtenido de $\mathrm{p}(\operatorname{sig})=0.000$ y es menor de 0.05 ; se rechaza la hipótesis nula y se acepta la alterna, demostrando que si hay influencia entre las variables independientes con la dimensión social.

De acuerdo con la hipótesis específica 3, establecimos una influencia de las variables violencia familiar y depresión en la dimensión familiar de la autoestima y se afirma que existe una influencia considerable al obtener un porcentaje de influencia de Nagelkerke del 62,9\%. Se obtuvo el valor de Chicuadrado de 5,403 que por ser menor al valor del Chi-cuadrado teórico de 21,03 se estableció que es el modelo adecuado para el ajuste de los datos. Así mismo esta influencia está reforzada por el valor de significancia obtenido de $\mathrm{p}(\mathrm{sig})=0.000$ y es menor de 0.05 ; se rechaza la hipótesis nula y se acepta la alterna, demostrando que si hay influencia entre las variables independientes con la dimensión familiar.

\section{DISCUSIÓN}

En el análisis que se realizó a los ítems de la prueba de violencia familia, se detectó que el 65\% de los encuestados afirmaron que cuando sus padres les gritan, ellos también hacen lo mismo; esto secunda la teoría de coerción de Patterson (como se citó en Cuenca y Mendoza, 2017), en la que los estilos de crianza inadecuados producen violencia, asimismo la teoría de transmisión intergeneracional, que sostiene que la familia influye en la tolerancia o ejercicio de la violencia.

Por otro lado, refuerza la referido en la teoría de aprendizaje social de Bandura (Boza, et al., 2017), en donde la agresividad se aprende por imitación de modelos violentos entre padres e hijos. Asimismo, lo hace con la teoría ecológica de Bronfenbrenner (como se citó en Hualpa, 2017) en cuanto la violencia es el resultado de la interacción con la familia, sociedad y cultura.

Nuestra investigación arrojó resultados en que el $24.3 \%$ de la muestra evidenció tener depresión severa y el $20 \%$ a nivel moderado, además de que las mujeres mostraban mayor puntaje en depresión severa a comparación de los varones; estos datos son similares con lo obtenido por Astocondor et al (2019) en donde la población que presentan mayor depresión son las de sexo femenino; así como con el estudio de Dostanic , et al (2021) en el que el 33.5\% padecía de depresión moderada y el 34.6\% tenía depresión severa. También concuerda con los resultados de Llanos y Ponce (2019) aunque en su investigación se obtuvo que el $76 \%$ mostraba depresión.

Refuerza también lo referido por Álvarez, et al., (2014 como se citó en Quispe, 2021), quienes manifestaron que las personas de sexo femenino tienden a padecer depresión debido a un conjunto de factores personales como cambios hormonales, su propia personalidad y culturalmente están en una posición vulnerable. Asimismo, desde la perspectiva de la filosofía existencial propuesto por Villegas (2021), el fallo ontológico se pone de manifiesto pues las mujeres cuestionan su existencia como personas 
valorables y consideran el quitarse la vida o al menos pensamientos de ese tipo, lo cual se evidenció en nuestra muestra en la que el 49\% ha tenido en algún momento ideación suicida.

Nuestro estudio, en el análisis de los ítems, obtuvimos que $44.2 \%$ de la muestra reconoce tener una pobre opinión acerca de sí mismo, el 50.7\% considera que físicamente no son atractivos, el $65 \%$ piensa que los demás son más valiosos y el $47.5 \%$ siente que le gustaría ser otra persona. Esto concuerda con el modelo de reconocimiento estructurado de Honneth (2010, como se citó en Pepe, et al., 2021) que sostiene que la humillación física, la exclusión social y la degradación son factores que afectan la autoestima de las personas. Lo mismo sucede con lo mencionado por Paucar y Barboza (2018) respecto a la presencia del componente cognitivo y afectivo en los resultados mencionados; además de la teoría humanista de Maslow (1991, como se citó en Vásquez, 2021) en la que el nivel de necesidad de reconocimiento se ha visto afectado.

Refuerza también la teoría de Rosenberg (1965, como se citó en Céspedes, 2020), en la que el componente afectivo y cognitivo respecto a su valoración y pensamientos sobre sí mismo, se ven alterados. La teoría de autovaloración de Cooley (como se citó en Panesso y Arango, 2017) y el modelo sociométrico de Leary y Baumeister (2000, como se citó en Flores, 2021) se implican en estos resultados ya que la muestra consideró que otras personas son más valiosas que ellos mismos por ende se ve afectado el valor que se tiene en función de la opinión de los demás.

Nuestro estudio halló una relación causal entre la violencia familiar y la depresión, resultados similares con las investigaciones de Rivera et al (2018) en la que el $41.4 \%$ de su muestra padecía de depresión además de hallar una asociación entre dicha variable con conflictos interparentales. El otro estudio concordante es el de Dostanic, et al (2021) en el que la violencia se correlaciona de manera significativa con la depresión y ansiedad, incrementando la probabilidad de ocurrencia si la agresión se presenta bajo los efectos del alcohol. Otra investigación que muestra resultados similares es la de Chahid, et al (2020), quienes demostraron que la autoestima, la ansiedad y la depresión influyen en el suicidio, entendida ésta como una violencia hacía sí mismo.

\section{CONCLUSIONES}

$\mathrm{Al}$ analizar las variables y contrastar la hipótesis general, se obtuvo un $\mathrm{p}=.00$ que es menor a .05 , por lo cual se rechazó la hipótesis nula y se determinó que existe influencia de la violencia familiar y la depresión sobre la autoestima en confirmantes de la Parroquia Jesús Vida y Paz del distrito de San Juan de Lurigancho, 2021. Además, el modelo presenta un buen ajuste y predice en un $60.6 \%$ la ocurrencia de las categorías de la variable dependiente frente a las independientes. 
$\mathrm{Al}$ analizar las variables y contrastar la primera hipótesis específica, se obtuvo un $\mathrm{p}=.00$ que es menor a .05 , por lo cual se rechazó la hipótesis nula y se determinó que existe influencia de la violencia familiar y la depresión sobre la dimensión sí mismo general de la variable autoestima en confirmantes de la Parroquia Jesús Vida y Paz del distrito de San Juan de Lurigancho, 2021. Además, el modelo presenta un buen ajuste y predice en un 58.7\% la ocurrencia de las categorías de la dimensión de la variable dependiente frente a las independientes.

$\mathrm{Al}$ analizar las variables y contrastar la segunda hipótesis específica, se obtuvo un $\mathrm{p}=.000$ que es menor a .05 , por lo cual se rechazó la hipótesis nula y se determinó que existe influencia de la violencia familiar y la depresión sobre la dimensión social de la variable autoestima en confirmantes de la Parroquia Jesús Vida y Paz del distrito de San Juan de Lurigancho, 2021. Además, el modelo presenta un buen ajuste y predice en un $35.2 \%$ la ocurrencia de las categorías de la dimensión de la variable dependiente frente a las independientes.

$\mathrm{Al}$ analizar las variables y contrastar la tercera hipótesis específica, se obtuvo un $\mathrm{p}=.00$ que es menor a .05 , por lo cual se rechazó la hipótesis nula y se determinó que existen influencia de la violencia familiar y la depresión sobre la dimensión familiar de la variable autoestima en confirmantes de la Parroquia Jesús Vida y Paz del distrito de San Juan de Lurigancho, 2021. Además, el modelo presenta un buen ajuste y predice en un $63.9 \%$ la ocurrencia de las categorías de la dimensión de la variable dependiente frente a las independientes. 


\section{REFERENCIAS}

Astocondor, J., Ruiz, L., \& Mejia, C. (2019). Influencia de la depresión en la ideación suicida de los estudiantes de la selva peruana. Horizonte Médico (Lima), 19(1), 53-58. https://dx.doi.org/10.24265/horizmed.2019.v19n1.09

Boza, N., Maldonado, F., Morales, M., y Meléndez, J. (2017). Violencia escolar, funcionalidad familiar y características sociodemográficas en una institución educativa pública de Huancayo-Perú. Revista Enfermería Herediana, 10(2), 123-130. https://doi.org/10.20453/renh.v10i2.3368

Chahid, H., Ahami, A., Chigr, F. \& Najimi, M. (2020). Depression, Anxiety, Self-esteem and Suicide Attempts: Study among High School Students in the Beni Mellal Region of Morocco. American Journal of Applied Psychology, 8(1), 9-15. http://dx.doi.org/10.12691/ajap-8-1-2

Céspedes, M. (2020). Violencia de género y autoestima en participantes de organizaciones sociales del distrito de Puente Piedra, 2020. [Tesis de maestría]. Universidad César Vallejo. Repositorio institucional. https://repositorio.ucv.edu.pe/handle/20.500.12692/55318

Cuenca, V. y Mendoza B. (2017). Comportamiento prosocial y agresivo en los niños: tratamiento conductual dirigido a padres y profesores. Acta de Investigación Psicológica. 7(2). 2691-2703. DOI: 10.1016/j.aipprr.2017.03.005

Díaz, V. (2017). Regresión logística y decisiones clínicas. Nutrición Hospitalaria, 34(6), 1505. https://dx.doi.org/10.20960/nh.1468

Diez, E. (2021). Eficacia de un curso de programación neurolingüística en la autoestima: Implicaciones de la programación neurolingüística en el aprendizaje. Revista de estilos de aprendizaje, 14(27). http://revistaestilosdeaprendizaje.com/article/view/2209

Dostanic, N., Djikanovic, B., Jovanovic, M., Stamenkovic, Z.\& Đeric, A. The Association Between Family Violence, Depression and Anxiety Among Women Whose Partners Have Been Treated for Alcohol Dependence. Journal o familiy violence. https://doi.org/10.1007/s10896-020-00238-1

Estrada, E., Gallegos, N., Mamani, H. y Zuloaga, M. (2021). Autoestima y agresividad en estudiantes peruanos de educación secundaria. Revista archivos venezolanos de farmacología y terapéutica. 40(1). http://doi.org/10.5281/zenodo.4675747

Fernández, A. (2019). Funcionamiento familiar en estudiantes de 2 do a 5to de secundaria con y sin depresión en una institución educativa privada de Villa El Salvador. [Tesis de licenciatura, Universidad Autónoma del Perú]. Repositorio institucional. http://repositorio.autonoma.edu.pe/handle/AUTONOMA/778

Flores, P. (2021). Relación entre estilos parentales y desarrollo de la autoestima en adolescentes. [Tesis de licenciatura]. Universidad de Lima. Repositorio institucional. https://repositorio.ulima.edu.pe/handle/20.500.12724/13211

Gonzales, L. (2017). La violencia familiar afecta la autoestima de los adolescentes en Lima-Perú. [Tesis de licenciatura, Universidad Católica Sede Sapientiae]. Repositorio institucional. http://repositorio.ucss.edu.pe/handle/UCSS/393

Hualpa, J. (2017). Violencia familiar y autoestima en estudiantes de 4to y 5to grado del nivel secundario en instituciones educativas públicas del distrito de Ate, 2017. [Tesis de licenciatura, Universidad César Vallejo]. Repositorio institucional. http://repositorio.ucv.edu.pe/handle/20.500.12692/11301?locale-attribute=en

Llanos, F. \& Ponce, C. (2019). Depresión y adherencia en personas afectadas con tuberculosis: Una exploración preliminar de datos. Revista de Neuro-Psiquiatría, 82(2), $104-109$. https://dx.doi.org/https://doi.org/10.20453/rnp.v82i2.3536 
Losada, A. V. y Caruso, J. M. (2021). Continuidad de los vínculos familiares: impacto de las resoluciones judiciales en violencia familiar. Revista de Psicología. https://dx.doi.org/10.24215/2422572Xe090

Ocampo, J., Guerrero, M., Espín, L., Guerrero, C., y Aguirre, R. (2017). Asociación entre índice de masa corporal y depresión en mujeres adolescentes. International Journal of Morphology, 35(4), 1547-1552. http://dx.doi.org/10.4067/S0717-95022017000401547

Panesso, K., y Arango, M. (2017). LA AUTOESTIMA, PROCESO HUMANO. Revista Electrónica Psyconex, 9(14), 1-9. https://revistas.udea.edu.co/index.php/Psyconex/article/view/328507

Paucar, M. y Barboza, S. (2018). Niveles de autoestima de los estudiantes de la institución educativa 9 de diciembre de pueblo nuevo chincha - 2017. [Tesis de licenciatura, Universidad Nacional de Huancavelica]. Repositorio institucional. http://repositorio.unh.edu.pe/handle/UNH/1900

Pepe, C., Moreno, M. y Martineli, G. (2021). Bienestar Social, Autoestima y Reconocimiento: Estudio Empírico sobre Crimen y Exclusión Basado en la Categoría de Menosprecio de Axel Honneth. Revista Colombiana de Psicología, 30(1). https://doi.org/10.15446/rcp.v30n1.80978

Quevedo, F. (2011). La prueba ji cuadrado. Medwave 11(12). http://dx.doi.org/10.5867/medwave.2011.12.5266

Quispe, C. (2012). Factores de riesgo asociados a depresión en estudiantes de enfermería de una universidad pública de Lima, 2021. [Tesis de licenciatura]. Universidad Nacional Mayor de San Marcos. Repositorio institucional. https://cybertesis.unmsm.edu.pe/handle/20.500.12672/16651

Radio Programas del Perú (2017, 05 de septiembre). San Juan Lurigancho es el distrito de Lima con mayor número de feminicidios. https://rpp.pe/lima/actualidad/san-juan-lurigancho-es-el-distrito-de-lima-con-mayor-indice-defeminicidios-noticia-1074660

Ramírez, G. (2019). La violencia familiar en el rendimiento académico de los estudiantes del nivel inicial. [Tesis de licenciatura, Universidad Nacional de Tumbes]. Repositorio institucional. http://repositorio.untumbes.edu.pe/handle/UNITUMBES/1936

Rivera, R., Arias, W. y Cahuana, M. (2018). Perfil familiar de adolescentes con sintomatología depresiva en la ciudad de Arequipa, Perú. Revista chilena de neuro-psiquiatría, 56(2). http://dx.doi.org/10.4067/s071792272018000200117

Rosas, A. (2019). Recursos didácticos y aprendizaje cooperativo de los estudiantes del Centro de Idiomas de la Universidad Cesar Vallejo de Lima. [Tesis de maestría] Universidad Nacional de Educación. Repositorio institucional. http://repositorio.une.edu.pe/handle/UNE/3588

Sánchez, A. y Farfán, E. (2019). Análisis Factorial Exploratorio del Inventario de Depresión de Beck (BDI-II) en universitarios cajamarquinos. Interacciones, 5(3), e177. https://doi.org/10.24016/2019.v5n3.177

Vásquez, C. (2021). Violencia familiar y autoestima durante la emergencia sanitaria por COVID - 19, en estudiantes de una institución educativa privada, Jaén, 2020. [Tesis de licenciatura] Universidad César Vallejo. Repositorio institucional. https://repositorio.ucv.edu.pe/handle/20.500.12692/62977

Villegas, M. (2021). Patologías de la libertad (V). Depresión y suicidio: La constricción del ser. Revista de Psicoterapia, 32(119). https://doi.org/10.33898/rdp.v32i119.86 